\title{
Modelling sterile insect technique to control the population of Anopheles gambiae
}

\author{
James E Gentile ${ }^{1 *}$, Samuel SC Rund ${ }^{2}$ and Gregory R Madey ${ }^{1}$
}

\begin{abstract}
Background: There is a renewed effort to develop novel malaria control strategies as even well-implemented existing malaria control tools may fail to block transmission in some regions. Currently, transgenic implementations of the sterile insect technique (SIT) such as the release of insects with a dominant lethal, homing endonuclease genes, or flightless mosquitoes are in development. These implementations involve the release of transgenic male mosquitoes whose matings with wild females produce either no viable offspring or no female offspring. As these technologies are all in their infancy, little is known about the relative efficiencies of the various implementations.

Methods: This paper describes agent-based modelling of emerging and theoretical implementations of transgenic SIT in Anopheles gambiae for the control of malaria. It reports on female suppression as it is affected by the SIT implementation, the number of released males, and competitiveness of released males.

Conclusions: The simulation experiments suggest that a late-acting bisex lethal gene is the most efficient of the four implementations we simulated. They demonstrate 1) the relative impact of release size on a campaign's effectiveness 2) late-acting genes are preferred because of their ability to exploit density dependent larval mortality 3) late-acting bisex lethal genes achieve elimination before their female-killing counterparts.
\end{abstract}

Keywords: Agent-based modelling, SIT, Release of insects with a dominant lethal gene (RIDL)

\section{Introduction}

Mosquito-borne illnesses including dengue fever, lymphatic filariasis (elephantiasis), yellow fever, and malaria make up $16 \%$ of the global disease burden, particularly so in the developing world [1]. Of these, malaria accounts for $18 \%$ of childhood deaths in sub-Saharan Africa [2] and in 2010 afflicted 219 million people and resulted in 660,000 deaths [3]. Malaria has been successfully controlled in many regions through vector-targeted intervention such as insecticide-treated bed nets (ITNs) and indoor residual sprays (IRS). However, these interventions will fail to eliminate malaria in regions with extremely high rates of parasite transmission and in areas where mosquito vectors are not susceptible to existing control techniques (such as by exophily or insecticide resistance) [4].

Sterile Insect Technique (SIT) is one control strategy that is gaining renewed interest for the control of mosquito populations [5-7]. The technique involves the

\footnotetext{
*Correspondence: jegentile@gmail.com

${ }^{1}$ University of Notre Dame, Cushing Hall, Notre Dame, USA

Full list of author information is available at the end of the article
}

mass release of males sterilized through radiological or chemical means. These mate with the wild population by out-competing non-sterile wild males [8]. Females mosquitoes (generally) mate only once, thus a successful mating with a sterile male will prevent the development of any offspring from the inseminated female $[9,10]$. In some insect pests such as the tsetse fly [11], medfly [12], and melon fly [13] SIT has proved enormously successful in achieving local control or elimination, including eradication of the screwworm from all of North America [14]. In mosquitoes, over two dozen SIT trials have been reported; however, issues such as poor competition with wild males, semi-sterility, or no ultimate adult population reduction - even despite successful sterile matings have been reported, reviewed in Benedict and Robinson 2003 [15].

Promising new advances in mosquito population control using the release of transgenic, instead of chemically or radioactively sterilized mosquitoes, are now garnering substantial interest [6]. These transgenic implementations are extensions of SIT, in that released males mate with 
wild-type females to abnormal results due to the males carrying a cell-lethal transgene. These implementations may allow for straightforward mass-rearing of a maleonly population for release; maintain larval competition with wild type mosquitoes; extend the "lifetime" of the intervention via propagation of the transgene through the population; and/or allow for the ability to induce or suppress the lethal trait through larval chemical exposure [16-19]. This paper broadly places these transgenic SIT implementations into one of four categories:

1. Early acting bisex (EBS) which is most similar to classical SIT whereby wild-type females mating with released males will produce no offspring. For modelling purposes, EBS is described as any implementation that involves the release of male mosquitoes modified such that no viable offspring (including larvae) are produced.

2. Early acting female-killing (EFK) whereby wild-type females mating with released males will produce no female offspring, but the transgene can be passed on through male progeny.

3. Late acting bisex (LBS) whereby wild-type females mating with released males will produce offspring that only survive through the aquatic stage and die shortly prior to or after emergence. Transgenic larvae that will eventually die prior to adulthood provide larval competition reducing wild-type larvae's chances of survival.

4. Late acting female-killing (LFK) whereby wild-type females mating with released males will produce offspring, but only male offspring survive to adulthood where they may propagate the transgene to their progeny. Transgenic female larvae that will eventually die prior to adulthood provide larval competition to wild-type larvae.

Release of insects carrying a dominant lethal gene (RIDL) is a transgenic implementation that has received the most recent attention. Thomas et al. and Heinrich et al., $[20,21]$ reported early success in the development of RIDL, generating strains of Drosophila with cell-lethal gene products under chemically repressible promoters expressed either in females only, or with female specific toxicity. Since then, the generation of two late acting RIDL strains of the dengue fever mosquito, Aedes aegypti, has been reported. This includes an EBS implementation with a repressible strain that kills all larvae, leaving no viable offspring [22]. This strain has been shown to compete reasonably well with the wild-type, with only a $5 \%$ reduction in survivability, a 4-day shortened average lifespan, and (perhaps beneficially) a one-day earlier emergence as an adult [23]. The other RIDL strain is a LFK implementation where adult females die immediately due to their inability to fly, whereas males remain to propagate the transgene [24]. RIDL strains are currently in trials, with early success reported in both large-cage [25] and field trials [26-28].

The success of SIT implementations is dependent on wild-type and mass-reared mosquitoes readily mating [29], although experience from mass-rearing campaigns of agricultural pests such as the screwworm has shown that significant loss of mating competitiveness can arise in mass-reared populations [30]. Some processes to sterilize mosquitoes (e.g. irradiation) can induce a reduction in mating competitiveness [31] but transgenic techniques can generate a line of sterile mosquitoes with no loss in mating competitiveness. This has been shown in Anopheles stephensi, Anopheles arabiensis, and Ae. aegypti when compared against the parent (non-transgenic) lab colonies [32-34]. However, when lab-reared transgenic Anopheles gambiae mosquitoes were compared in large-cage field trials against wild collected mosquitoes, reductions in mating competitiveness were indeed noted, although competitiveness was still better than those achieved and accepted for use in the medfly control programs [35]. Taking into account the mating competitiveness of transgenic-mass reared mosquitoes is therefore another important consideration to make when planning or considering the implementation of an SIT campaign, especially when determining the mosquito release size which may need to be increased to counteract reduced competitiveness.

Much work on the malaria mosquito, An. gambiae, remains to be done. However, there are early and promising successes. Recent work developing mosquitoes carrying homing endonucleases (HEG) has shown progress $[17,36]$. Various implementations of homing endonucleases work by selectively destroying the $\mathrm{X}$ chromosomepreventing female offspring (an EFK implementation) or genes vital to males and/or females. Additionally, Thailayil et al., [37], successfully demonstrated an EBS implementation using RNAi to knockdown the production of sperm.

This manuscript reports the first use of agent-based modelling to evaluate four implementations for the control of An. gambiae populations through the release of transgenic male mosquitoes at various release proportions and mating competitiveness rates. This work complements previous efforts to model SIT implementations which are summarized in Table 1. Agent-based modelling is used to simulate frequent releases of male transgenic mosquitoes homozygous for a cell-lethal transgene. The transgene exclusively kills the intended individuals $100 \%$ of the time (i.e. LFK will kill no males, kills all females, and no early larvae die). The results provide additional evidence that transgenic implementations of SIT could be used with success to eliminate An. gambiae vector populations, and estimate the relative success of various implementation strategies. 
Table 1 A survey of SIT modelling literature

\begin{tabular}{lll}
\hline [38] Foster et al. 1988 & Biological observation & Model notes \\
\hline & Modelled EBS and female-killing of a & Computational model that works on \\
& hypothetical insect population at various \\
& migrations, release rates, incomplete sterilities, \\
& and number of mutated alleles. Under most, \\
but not all scenarios, EBS achieves better & discrete generations comparing each male \\
genotype with each female genotype. & \\
\hline [39] Schliekelman and Gould 2000a & The authors model a hypothetical transgenic & \\
& implementation in hypothetical insects & The model uses combinatorics to determine \\
& whereby there are multiple lethal genes & a population's genetic make-up as inherited \\
& in released insects and these lethal genes \\
are conditional, killing only when certain & from parents. Lethality is operational in a \\
population subset with the correct allele & active in their genotype.
\end{tabular}

conditions are met and otherwise propagate.

Found that under ideal conditions, this

implementation can be far more effective

than traditional EBS.

[40] Schliekelman and Gould $2000 \mathrm{~b}$
Modelled transgenic implementation whereby

2-20 lethal genes were engineered into a

hypothetical insect. As the number of lethal

genes per released animal increases, there is a

greater chance any one progeny will inherit a

lethal gene. Found under ideal conditions,

control could be achieved at rates several

orders of magnitude more effectively than

single gene EBS.

$\begin{array}{ll}\text { [41] Barclay } 2001 & \text { Modelled EBS in hypothetical insects, with } \\ & \text { special regard to incomplete sterility and lack } \\ & \text { of competitive mating ability, which cause } \\ & \text { decreased levels of control success. }\end{array}$

[42] Esteva and Yang 2005

[22] Phuc et al. 2007

[44] Kean et al. 2008

[45] Yakob et al. 2009

[46] White et al. 2010
Models EBS implementation in males engineered to have no sperm. Release proportion is important.

Compared EBS to LBS. They found that EBS at low release ratios can increase equilibrium size of adult population, but LBS can result in eradication. At high release ratio EBS works but LBS works better.

Frequent small releases of EBS moths may be more effective than less frequent releases. They also compared how competitiveness of irradiated males effected control. Models doses of radiation which result in reduced, but not increased mating competitiveness.

\section{Modelled LBS, EBS, EFK, and LFK of a} hypothetical insect population at various release proportions, migrations, density dependancies, and fecundities. Found bisex lethal could be preferred over female killing under certain scenarios.

Models Ae. aegypti, EBS and LBS releases. Found control is more effective with fewer males released more often than many males released less frequently. complete sterilisation of males to the benefit of
The analysis is performed with a discretetime population model. The paper reports on many factors including equilibrium female population with regards to incomplete fertility.

Equation-based population model with density dependent mortality.

Time-delayed difference equation model with a density-dependent mortality in the aquatic life-stage and based on [43]. The difference between EBS and LBS was characterized in population suppression.

Discrete-time population model with overlapping generations. This model takes into account an over flooding parameter and incomplete sterility.

Time-delayed difference equation model representing the mosquito's lifecycle with adult and larval mortality terms.

Population dynamics are modelled with a time-delayed difference equation model extended from [43]. EBS and LBS are modelled and the dynamics of injected pulses of mosquitoes are reported. 
Table 1 A survey of SIT modelling literature (Continued)

\begin{tabular}{|c|c|c|}
\hline [47] Deredec et al. 2011 & $\begin{array}{l}\text { Models an An. gambiae EFK implementation } \\
\text { where the X chromosome in sperm is targeted } \\
\text { (and two other transgenic techniques that are } \\
\text { outside the scope of this paper) by release } \\
\text { of mosquitoes carrying homing endonuclease } \\
\text { genes (HEG). Determined the number of } \\
\text { individual HEGs targeting essential mosquito } \\
\text { genes required at various mosquito } \\
\text { reproductive numbers with various homing } \\
\text { rates to eliminate a mosquito population. }\end{array}$ & $\begin{array}{l}\text { This work extends a population model } \\
\text { by adding HEG dynamics and focuses on } \\
\text { reducing the intrinsic reproductive rate of } \\
\text { the female population. Density dependent } \\
\text { mortality is considered for larvae. }\end{array}$ \\
\hline [37] Thailayil et al. 2011 & $\begin{array}{l}\text { Models release size of spermless An. gambiae } \\
\text { (EBS) males required at differing rates of } \\
\text { occurrences where females mate more than } \\
\text { once. Very low levels of remating events were } \\
\text { found to have significant negative effects on } \\
\text { the ability to control the mosquito population. }\end{array}$ & $\begin{array}{l}\text { Differential equation model with no explicit } \\
\text { time latency between generations. The } \\
\text { adult female population separated into } \\
\text { females who have not mated; mated and } \\
\text { fertile; mated; and infertile. Population } \\
\text { persistence was described in terms of the } \\
\text { model coefficients. }\end{array}$ \\
\hline [48] Dumont and Tchuenche 2011 & $\begin{array}{l}\text { Found it more effective to have small and } \\
\text { frequent releases of EBS males over large } \\
\text { infrequent releases. Also EBS works better } \\
\text { when carried out with a larval habitat control } \\
\text { program (mechanical control). }\end{array}$ & $\begin{array}{l}\text { Extensive system of equations which } \\
\text { captures population and compartmental } \\
\text { dynamics. }\end{array}$ \\
\hline [49] Lee et al. 2013 & $\begin{array}{l}\text { Modelled EBS \& LBS in Ae. aegypti mosquitoes } \\
\text { under endemic and emerging outbreak } \\
\text { scenarios. Evaluated various release and } \\
\text { intervention-region sizes. Found EBS was } \\
\text { always more effective than EBS, though the the } \\
\text { magnitude varied by situation. }\end{array}$ & $\begin{array}{l}\text { Difference equation model similar to [22] } \\
\text { but look at an endemic case and emerging } \\
\text { outbreak of mosquito populations. }\end{array}$ \\
\hline
\end{tabular}

\section{Methods}

Four hypothetical SIT implementations were evaluated with an agent-based model for An. gambiae. Virtual mosquito agents traversed states that characterized many behavioural and life-stage aspects of the mosquito. Development was determined by a virtual mosquito's own attributes, namely sex and genotype. All four SIT implementations were represented as a cell-lethal gene expressed in the virtual agents. For EBS, these dynamics can be equivalent to methods that render males with non-viable sperm. Agent-based modelling may be a preferred approach to simulate SIT implementations because it allows us to characterize individual mosquitoes, encode the effect of various genotypes, and model the effects of overlapping generations.

The mosquito agent's states are represented in Figure 1 while Table 2 outlines the transition rules. Mosquito agents entered the model as eggs and emerged as adults after transitioning through the larval and pupa states. Immature adults developed then sought a mate (females were randomly mated with a mate-seeking male). Males remained mate-seeking while mated females began to complete the gonotrophic cycle. A new agent was created for each egg deposited by an adult female. This agentbased model is fully described in [50] but this section will highlight elements pertaining to the population dynamics where SIT methods can have an important effect.
Egg and pupa state time durations are described as 24 hours plus a 'hatch-time' $\left(H_{t}\right)$ term. $H_{t}$ was designed to reflect observations that most eggs hatch within three days, however, some take as long as five days [51]. Hourly $H_{t}$ determines the amount of time an agent takes to hatch and is designed as a piecewise function of $x$, a variable sampled from a uniform distribution, $U(0,1)$, and is defined as:

$$
H_{t}(x)= \begin{cases}40 \cdot x & : x \leq 0.5 \\ 68.57 \cdot x-10.28 & : 0.5<x \leq 0.85 \\ 480 \cdot x-360 & : 0.85<x \leq 0.9 \\ 600 \cdot x-468 & : 0.9<x \leq 0.94 \\ 2400 \cdot x-2160 & : 0.94<x \leq 1.00\end{cases}
$$

and seen in Figure 2.

Mortality was experienced differently at each life stage: eggs and pupae were subject to a fixed daily mortality rate of 0.1 ; adult mortality rate $\left(M_{\text {adult }}\right)$ was age dependent described as

$$
M_{\text {adult }}(n)=\frac{0.1 \cdot e^{n / 25}}{1+0.25\left(e^{n / 25}-1\right) .}
$$

deriving from [52] where $n$ is the age in days. The larva mortality rate $\left(M_{\text {larva }}\right)$ is density dependent favouring older agents

$$
M_{\text {larva }}(n)=0.1 \cdot e^{\frac{L_{\text {mass }}}{n \cdot C}}
$$




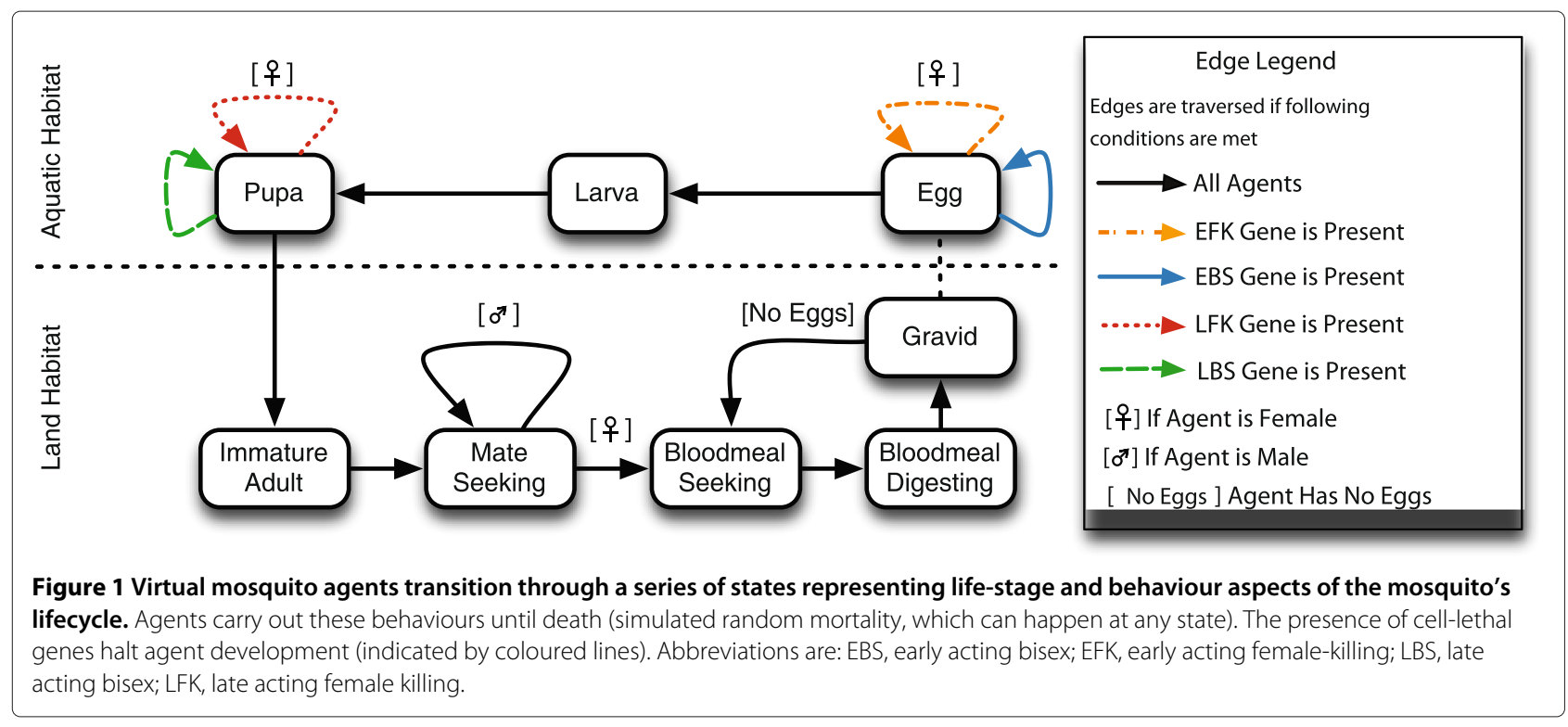

where $C, L_{\text {mass }}$, and $n$ are the larval habitat's carrying capacity, larval biomass and larva age in days, respectively. The larval biomass, $L_{\text {mass }}$, is an age-weighted sum of the larva population:

$$
L_{\text {mass }}=\sum_{n=1}^{10} n \cdot L_{n}
$$

$L_{n}$ is the number of $n$ day-old larvae, these equations characterize larval growth as linear with daily age. Larval mortality is largely controlled by the $L_{\text {mass }} / C$ term in Equation 3. Assuming a constant $C$, larval mortality increases as larval biomass in the habitat $\left(L_{\text {mass }}\right)$ increases. A larval habitat with fewer larvae will allow for a higher survival rate. This is analogous with previous models [43] but also favours older larvae.
A mosquito agent's sex and genotype were assigned when it is created. Sex was randomly assigned with an equal probability of male and female. Each agent has two alleles of each gene where one was randomly contributed from the mother and the other was from the father. Celllethal genes were dominant and manifested if present. EFK carrying females stopped development in the egg state and EFK carrying males developed as normal. EBS and LBS expressing agents ceased developing in the egg and pupa states respectively.

Mate-seeking females were assigned a mate at random from a distribution of all mate-seeking males weighted by each male's respective competitiveness values. Competitiveness represents an agent's ability to mate relative to a true wild-type (if this value is $50 \%$, it is half as competitive as wild-types). Every agent had a mating competitiveness

Table 2 The state transition rules of the model

\begin{tabular}{llll}
\hline State & Duration & Exit condition & Note \\
\hline Egg & 1 day $+H_{t}$ & None & Reflects incubation and hatch time. \\
\hline Larva & about 12 days & Nighttime & $\begin{array}{l}\text { Larval mortality is density dependent and favours } \\
\text { older larvae. }\end{array}$ \\
\hline Pupa & 1 day $+H_{t}$ & Nighttime & $\begin{array}{l}\text { Adult emergence from pupae occurs (6 P.M. to 6 A.M. } \\
\text { in the simulation). }\end{array}$ \\
\hline Immature Adult & 53 hours & None & Mating is 100\% successful and mate is assigned randomly. \\
\hline Mate Seeking & - & 6 P.M. \& Female & Females have a 25\% chance of finding a host each hour. \\
\hline Bloodmeal Seeking & - & Meal Success \& Nighttime & Bloodmeals take less than 1 hour. \\
\hline Bloodmeal Digesting & 36 hours & Nighttime & Agents seek to lay eggs in larval habitats only at night. \\
\hline Gravid & - & Empty Egg Clutch \& Nighttime & Agents complete gonotrophic cycles until death. \\
\hline
\end{tabular}

The model has an hourly time resolution and many agent behaviours are dependent on the time in state and simulated time of day. $H_{\mathrm{t}}$ is a randomly assigned 'hatch time' value described in Equation 1. 


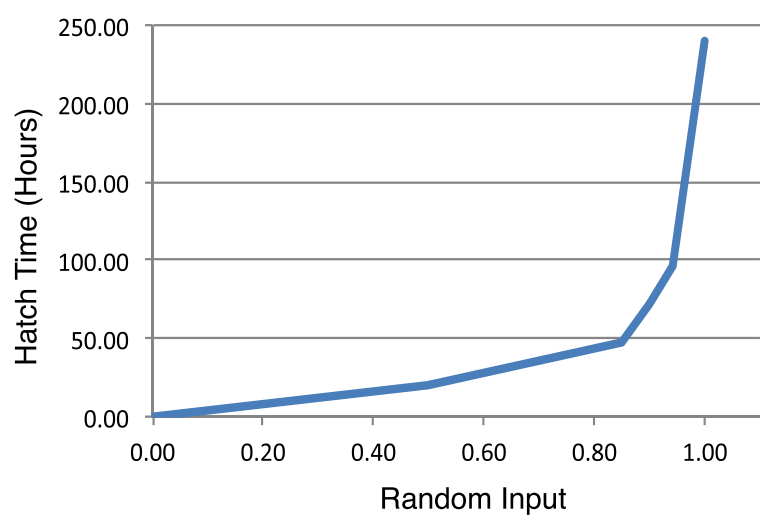

Figure 2 Visualization of the hatch-time curve given a random input value.

attribute determined by averaging the values of its mother and father. Female competitiveness did not affect mating probability but was considered when determining the female mosquito's progenies' values.

\section{Simulated SIT Campaigns}

The simulations captured one year of intervention with an hourly time resolution. A steady agent population persisted for six months prior to the intervention period and an average male population was obtained through 30 consecutive daily observations. Next, homozygous, mateseeking males were released into the simulation every day for one year. Released males' mating competitiveness was defined with a simulation parameter. Releases occurred at 3 P.M. and the release number was a fixed amount obtained by multiplying a release proportion parameter and the average wild-type male population before the releases started (this was rounded to the nearest whole number). Note the release number remained the same even if the adult male population was being suppressed.

The modelling approach used in this paper allowed us to record adult and larval population numbers and values within the simulation that contribute to the population dynamics. These numbers were normalized and populations were stratified by sex and genotype. The simulation recorded a fecundity potential measure which relates to the probability that a wild-type female adult will have wild-type female offspring. Given a set of male mosquitoes $\left(\mathbf{x}_{i} \in X\right)$ each has a mating competitiveness $\left(m_{i}\right)$, and is wild-type, heterozygous or homozygous $(\mathbf{x} \in$ $\left.\left\{\left[\begin{array}{lll}1 & 0 & 0\end{array}\right],\left[\begin{array}{lll}0 & 1 & 0\end{array}\right],\left[\begin{array}{lll}0 & 0 & 1\end{array}\right]\right\}\right)$. Fecundity potential $(F)$ is defined as

$$
F=1-\frac{\sum m_{i} *\left[\begin{array}{lll}
0 & 0 & 1
\end{array}\right] \cdot \mathbf{x}_{i}}{\sum m_{i}}-\frac{\sum m_{i} *\left[\begin{array}{lll}
0 & 1 & 0
\end{array}\right] \cdot \mathbf{x}_{i}}{2 \cdot \sum m_{i}} .
$$

The first and second numerators return the total mating competitiveness of homozygous and heterozygous males respectively. The coefficient in the second denominator accounts for the fact that half of the female progeny from a wild-type animal mating with a heterozygous animal will carry the cell-lethal gene.

Finally, the simulation reported the mortality term $L_{\text {mass }} / C$ which is proportional to the density dependent mortality experienced by larvae in Equation 3. Measures were normalized by dividing their values with the output given no SIT campaign (population values were normalized using wild-type numbers).

\section{Results}

SIT campaigns were simulated varying the cell-lethal gene implementation, number of released males, and the mating competitiveness of released mosquitoes. A campaign's effectiveness was measured in terms of wild-type female adult population suppression as this term relates to the transmission coefficient of vector-borne illnesses. Recall the simulation reached a steady population for 6 months and an average male population was obtained from 30 consecutive daily observations. Then the daily releases started and the number of released males was the release proportion multiplied with the average male population. All reported results are an average of 30 simulations since the models in this paper are stochastic.

Plots show measures in terms of release proportion and time after normalising them with average measures given no interventions. Figure 3 is time-series plots showing the simulation behaviour at all time-steps for all populations but with one release proportion. Proportion-series plots show an average measure over 30 consecutive simulated days (Figure 4). Figure 5 demonstrates population suppressions over time for each gene with all release proportions. There are ten measures indexed with Roman numerals on Figure 3 where $L$ represents a cell-lethal transgene. Measures are:

i) $L^{(-/-)}$Adult Females: population of wild-type adult females.

ii) $L^{(-/-)}$Adult Males: population of wild-type adult males.

iii) $L^{(+/+)}$Adult Males: population of released males.

iv) $L^{(-/+)}$Adult Males: population of males heterozygous for the cell-lethal gene.

v) Fecundity Potential: value related to the chance that a female will have wild-type female offspring.

vi) $L^{(-/-)}$Larval Females: population of wild-type larval females.

vii) $L^{(-/-)}$Larval Males: population of wild-type larval males.

viii) $L^{(-/+)}$Larval Females: population of larval females heterozygous for the cell-lethal gene.

ix) $L^{(-/+)}$Larval Males: population of larval males heterozygous for the cell-lethal gene. 


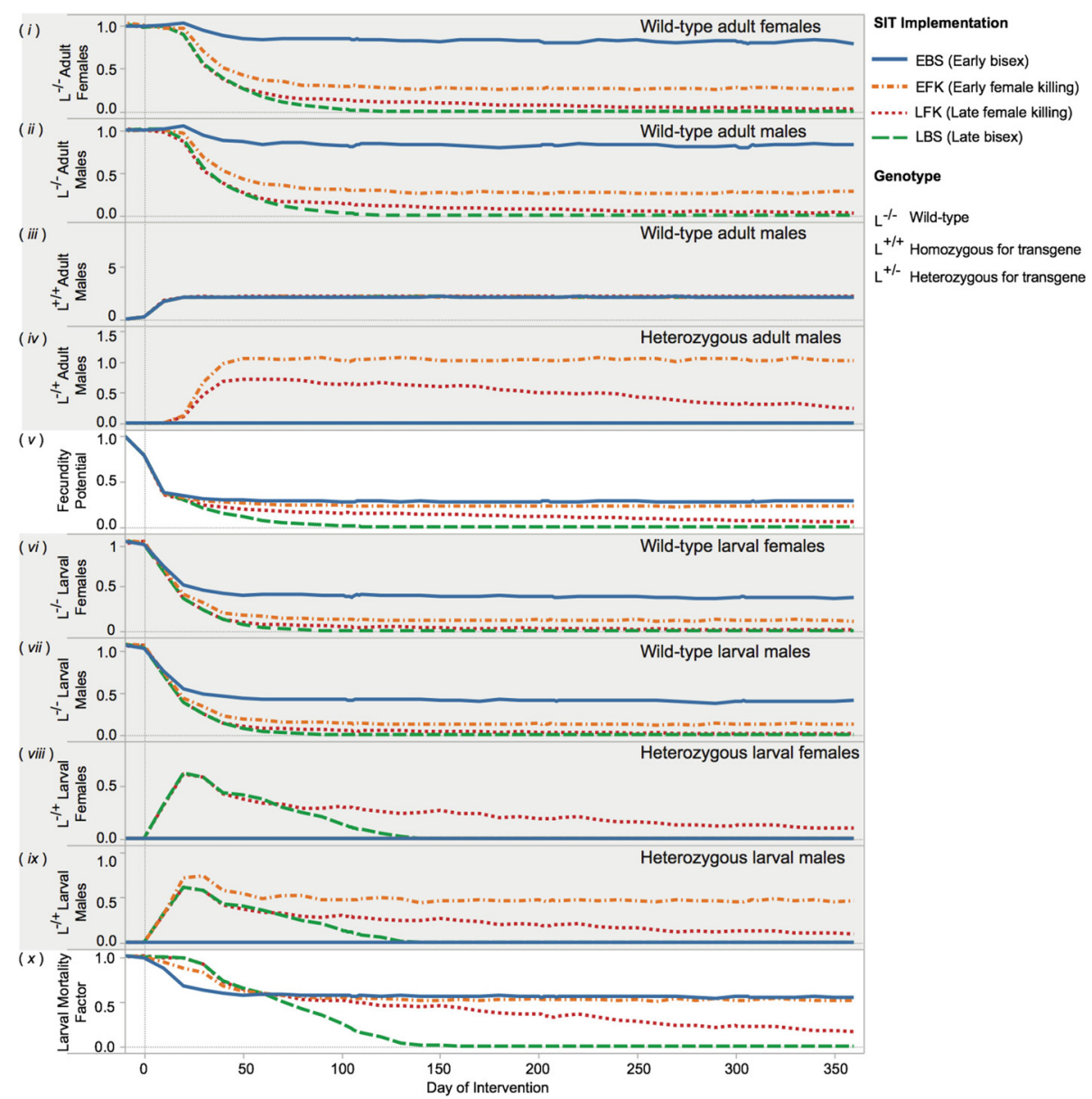

Figure 3 Simulated daily, fixed-number releases of sterile males with SIT genes that halted growth in all eggs (EBS), only female eggs (EFK), all pupae (LBS), or female-only pupae (LFK). These plots report the population's response to the introduction of sterile males at the release proportion of 0.3 from the first release of the campaign (day 0). Released males are as competitive as wild-types in these graphs. From top to bottom, these measures are the number of wild-type adult females (i) and males (ii); number of homozygous and heterozygous adults males (iii and iv); the fecundity potential ( $v$ ); wild-type female and male larvae ( $v$ i and vii); heterozygous female and male larvae (viii and $i x$ ); and the density-dependent larval mortality factor $(x)$. $L$ represents the cell-lethal transgene. Please note that mosquitoes are counted before releases but the fecundity potential measure is calculated after.

x) Larval Mortality Factor: value proportional to the density-dependent larval mortality.

The population dynamics are discussed in terms of initial and final effects. Initial effects detail the population's response to releases of males with the cell-lethal gene. Final effects are measures in the 12th month of a simulated year-long campaign. Final effects are determined to be important so the discussion is tailored thusly.

\section{Initial effects}

First, the mosquito population's initial response to the release of lab-reared males in a simulated SIT campaign is considered. Figure 3 shows an example of the response to an SIT release with the release proportion of 0.3 .
The initial effects, unless otherwise noted, were largely independent of the release proportion.

Several responses were expected given the biological mechanisms that the model captures. Wild-type adult population suppression would not be seen until females mated with a released male, acquired a bloodmeal, developed a clutch of eggs, and her progeny reached adulthood (about 17 days). At this time, heterozygous adult males should first appear for FK methods and the effect on larval mortality would not be observed until the progeny of wild-type mosquitoes mating with released mosquitoes are expected to be larvae (about 5 days) and heterozygous larvae should appear.

These expectations are clearly shown in the charts in Figure 3. Plots $i$, iii, and $i v$ show the delay from the first 


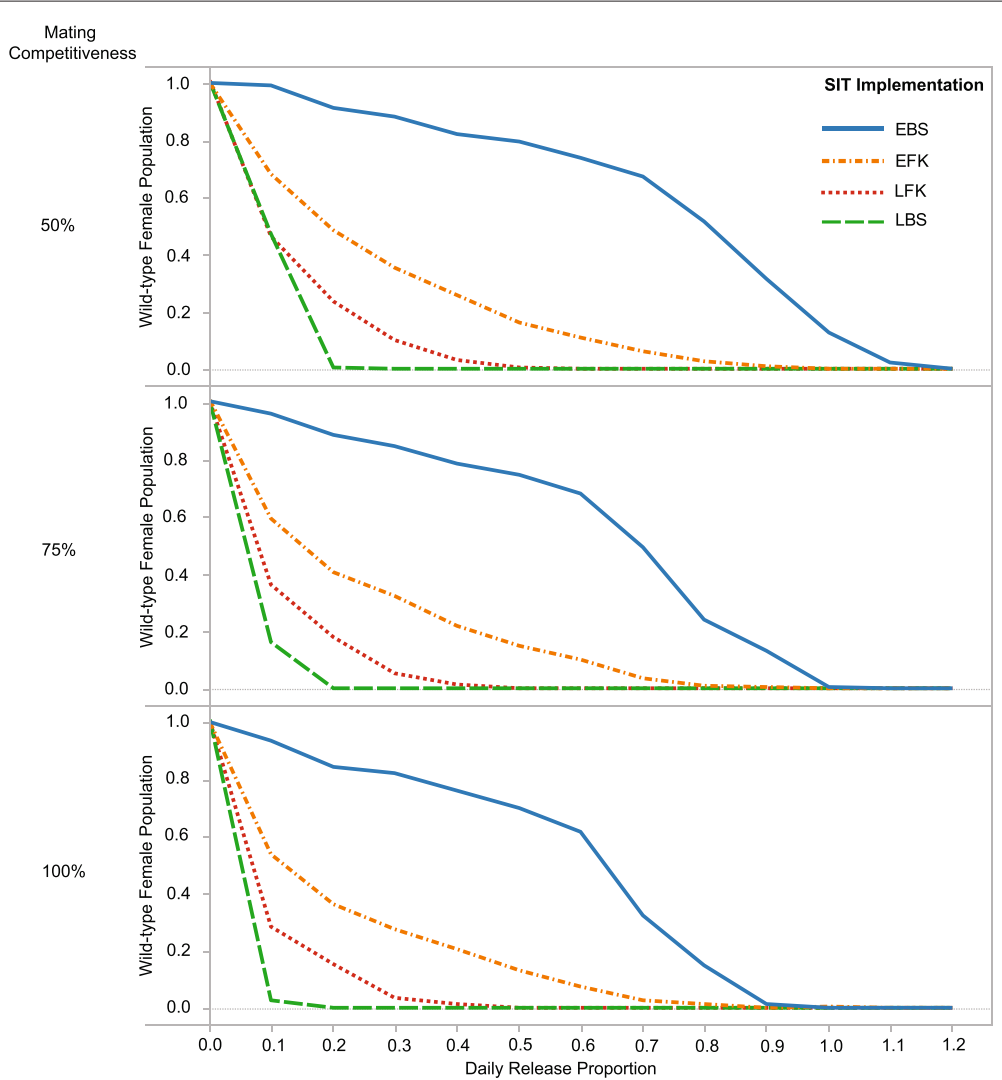

Figure 4 Population suppression factor reported in terms of released mating competitiveness and daily release proportion for the SIT implementations in the 12th month of the campaign. A suppression factor of 0.5 means the wild-type population was halved relative to a population with no intervention employed. Competitiveness represents a male agent's ability to mate relative to a true wild-type male (if this value is $50 \%$, it is half as competitive as wild-types).

release to evident population suppression and the appearance of heterozygous males. The aquatic population's responses are seen in $v i, v i i$, viii, $i x$, and $x$ and demonstrate the delayed effect of releases on larval mortality.

The injection of a cell-lethal gene can disrupt the wildtype population's equilibrium and this is especially seen in EBS methods where there is a brief, minor increase in wild-type populations (this is seen around day 20 in Figure 3 ). This is likely due to less density dependent mortality in the larval habitats allowing a few more agents to emerge but this effect is immediately followed by population suppression. Other models have shown that cell-lethal genes can cause an increase in the wild-type population [22]; in the results, this effect is very short.

\section{Final effects}

Whereas the initial effects are largely independent of release proportion, a high release proportion is crucial to population elimination one year following SIT release (Figure 3). Late-acting genes are far more effective than their early-acting counterparts because of their ability to maintain higher larval mortality. Finally, bisex cell-lethal genes are more effective with late-acting implementations.

\section{Release proportion}

A campaign's effectiveness is largely dependent on the number of consistently released males on a daily basis. Figure 4 demonstrates that greater population suppression occurs if more sterile males are released. However, there appears to be a point where releasing more sterile males does not cause a noticeable benefit.

Figure 5 shows there is little benefit to releasing LFK males at a higher proportion than 0.7. It is likely that a point of diminishing returns exists for this cell-lethal gene. Though these points are not evident in all the lines of the figure, it is reasonable to assume they exist for all transgenic implementations (assuming a high enough release proportion). Operating above this point could be a waste or misappropriation of resources. 


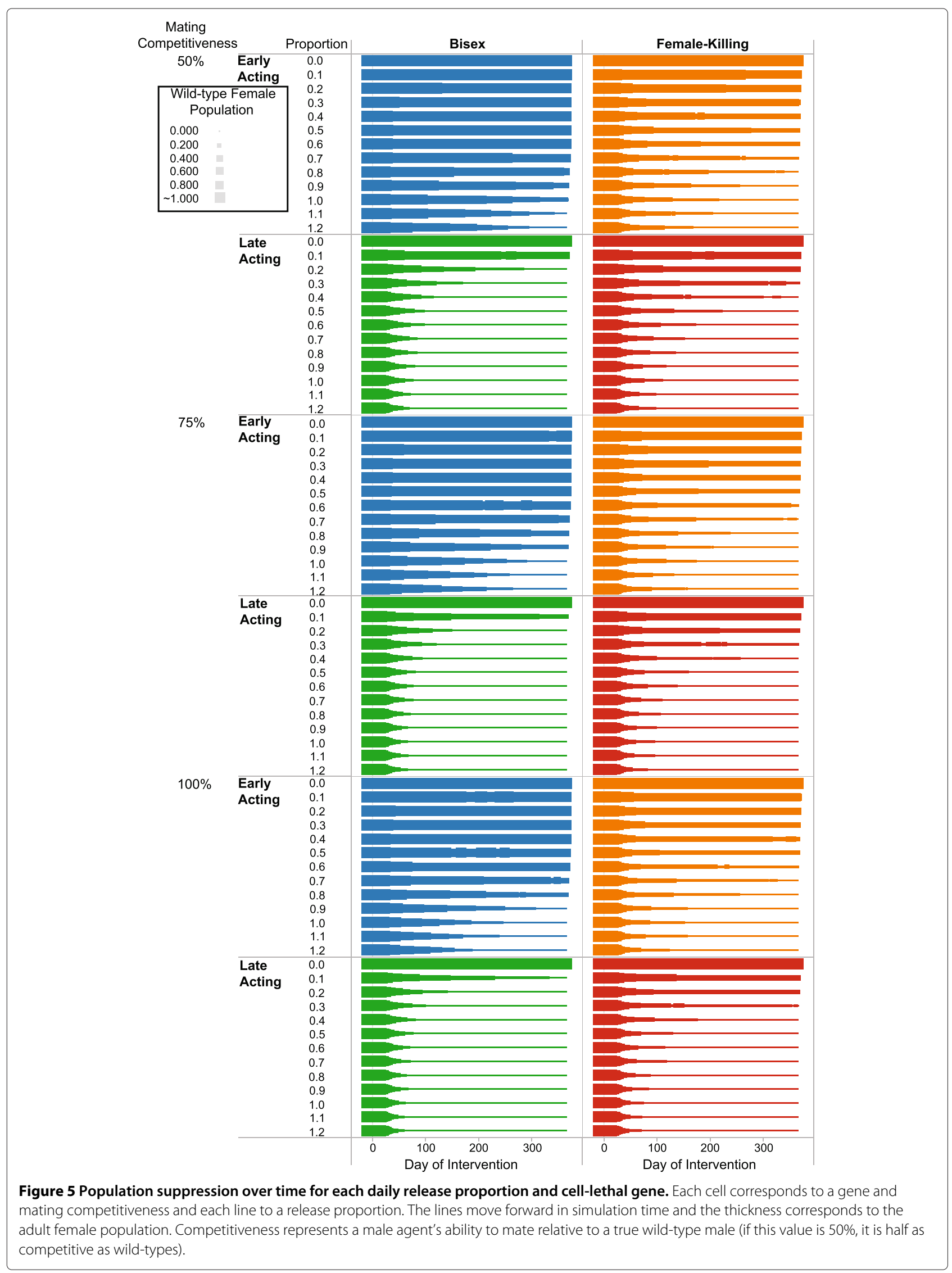




\section{Early-acting versus late-acting}

It may be reasonable to hypothesize that late-acting SIT implementations are efficient at suppressing the population because they exploit density dependent mortality in the larval habitat. This principle is observed in the results as well. Late-acting genes achieved elimination with fewer released males when compared with their corresponding early-acting counterparts. Early in the campaign, lateacting genes caused the larval mortality to remain higher and this is seen in Figure 3 Plot $x$. This caused more wildtype population suppression and an eventual decrease in larval mortality.

\section{Mating competitiveness}

The effectiveness of an SIT campaign can be reduced if released males are less competitive when mating. The suppression factor is reported for the last 30 days of a 12 month SIT campaign in terms of daily release proportion and mating competitiveness in Figure 4. In these plots, it is clear that LBS still performs better than LFK because eradication is achieved with the lowest proportion of released males.

The effects of mating competitiveness are less apparent on female-killing methods. This is likely because second generation heterozygous males serve as an additional suppression source and these males are more competitive than homozygous mosquitoes.

\section{Female-killing versus bisex lethality}

The late-acting, bisex, cell-lethal implementation achieved eradication with the fewest number of released males. Late-acting, female-killing genes are often thought to be very effective because they generate a population of males which serve as an additional reservoir for the celllethal gene. These results indicate that this population serves as a reservoir for the wild-type gene (illustrated in Figure 6) and reduces a campaign's effectiveness. This point can be observed in Figure 3 by comparing the fecundity potential $(v)$ in relation to heterozygous males $(i v)$ and the persistence of the wild-type female population $(i)$.

For the early-acting methods, female-killing genes outperformed bisex cell-lethal implementations. EFK allowed for the presence of sterile male larvae and these agents contributed to the density dependent mortality in the larval habitat. That effect was primary in population suppression and was not overcome by the heterozygous male population acting as a reservoir for the cell-lethal gene.

\section{Discussion}

This paper used agent-based modelling to investigate the effect of four SIT implementations on a simulated $A n$. gambiae population. The simulations represented daily, fixed-number releases of sterile males with SIT cell-lethal tannsgenes that halted growth in all eggs (EBS), only female eggs (EFK), all pupae (LBS), or female-only pupae (LFK). In the female-killing methods (EFK, LFK), a heterozygous male population survived and could pass a celllethal gene to future generations. The lab-reared mating competitiveness was varied along with the male release proportion. The cell-lethal genes were assumed to be $100 \%$ effective at only one locus. The conclusions are as follows:

- Population suppression is dependent on the number of released males in a campaign for any cell-lethal gene to a point. After a certain release proportion, the additional males appear to have diminishing returns on population suppression likely due to saturation of the cell-lethal gene. This result is congruent with previous results $[44,46,48]$ but this work shows the dynamic is present with all four cell-lethal implementations tested and using an agent-based modelling technique.

- Late-acting cell-lethality is highly preferred over

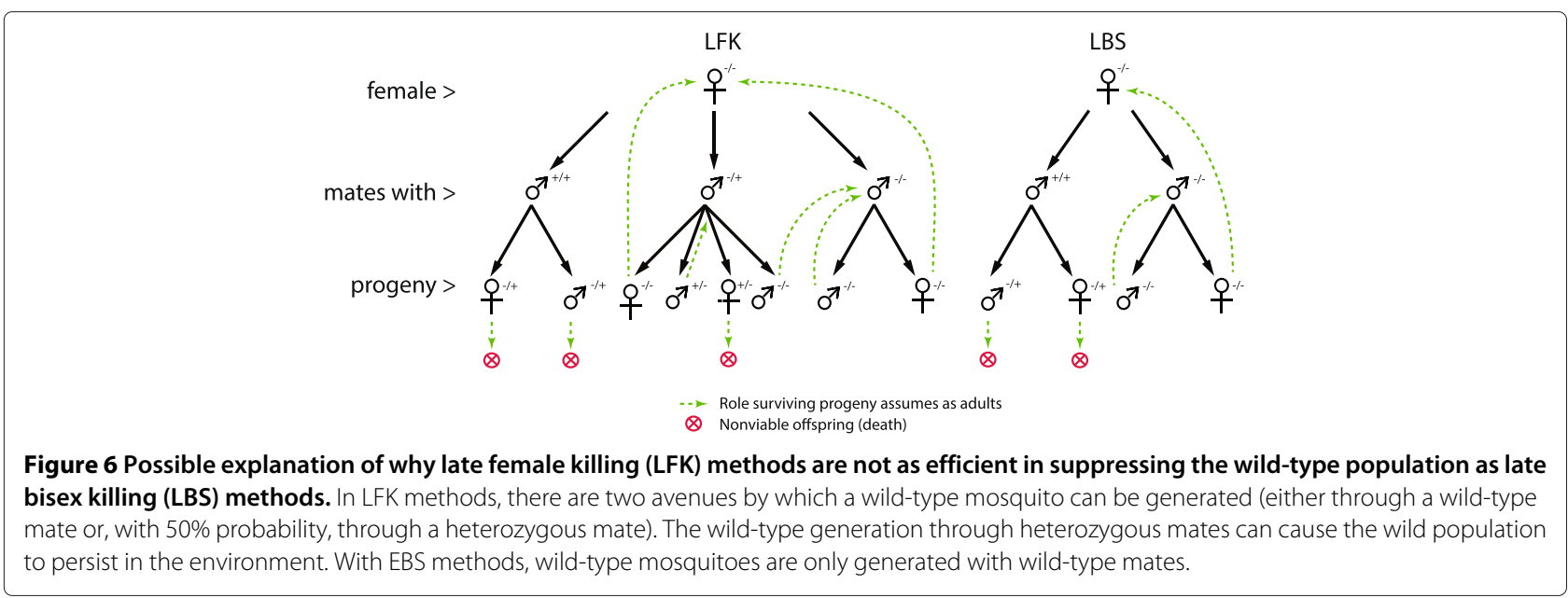


early-acting because of its ability to exploit densitydependent mortality in the aquatic habitat. A large portion of population suppression occurs within 50 days of the campaign's beginning. Late-acting genes maintained a higher larval mortality leading to greater population suppression by the end of a simulated year-long campaign. These results are also congruent with previous modelling literature $[22,53]$ but they are demonstrated in an Anopheline simulation.

- Late-acting bisex genes are preferred because these genes lead to mosquito elimination with the fewest number of released males. This is a dynamic in SIT systems and may be counterintuitive though presented in [38]. Female-killing methods are thought to be more efficient because the heterozygous male population is an additional reservoir for the cell-lethal gene. However, the converse is that this population can also serve as a reservoir for wild-types and cause a population to persist (this is illustrated in Figure 6).

The findings in this manuscript are not without limitations. For example, using current technologies, 100\% lethality is not always ensured, but this is not modelled; instead assuming that $100 \%$ lethality will be favoured over sub-optimal mating competition. Further, lethal genes could be introduced at multiple loci, which could increase efficiency considerably. Additionally, only males are assumed to be released and no females are released due to sorting difficulties. Clearly, successful female killing strategies would prevent such accidental release of females. Finally, seasonality is not modelled, assuming constant temperatures and larval habitat carrying capacity.

This paper used an agent-based model to simulate four transgenic sterile insect technique implementations and provide feedback on their efficiency in suppressing a mosquito population. Three conclusions were reached from the system's emergent behaviour; two were in agreement with the previously published literature and one is a novel insight which has not yet seen reported. These results demonstrate the potential of agent-based modelling to validate with other results but also expose novel, emergent behaviour in a complex system.

\section{Competing interests}

The authors declare that they have no competing interests.

\section{Authors' contributions}

JEG designed the computer simulations and experiments. All authors contributed to the interpretation of results and authoring of this manuscript. All authors read and approved the final transcript.

\section{Acknowledgements}

We would like to thank Dr. Mark Q. Benedict for his review of this work. A special thanks to Chris Frederick and Jim Leisring at the Office of Information Technologies at The University of Notre Dame for their consultation on various data warehouse and reporting technologies. We also acknowledge the Center for Research Computing's Cluster at The University of Notre Dame. S.S.C.R. is funded by a strategic award from the Wellcome Trust for the Centre for Immunity, Infection and Evolution (grant no. 095831).

\section{Author details}

${ }^{1}$ University of Notre Dame, Cushing Hall, Notre Dame, USA. ${ }^{2}$ Centre for Immunity, Infection and Evolution, School of Biological Sciences, University of Edinburgh, Edinburgh, UK.

Received: 8 September 2014 Accepted: 26 January 2015

Published online: 22 February 2015

\section{References}

1. World Health Organization. World Health Report 2004: Changing history. 2004. http://www.who.int/whr/2004/en/. Accessed 02 February 2014.

2. Bryce J, Boschi-Pinto C, Shibuya K, Black R. WHO estimates of the causes of death in children. Lancet. 2005;365:1146-52.

3. World Health Organization. World Malaria Report 2012. 2012. http://www. who.int/malaria/publications/world_malaria_report_2012/en/. Accessed 02 February 2014.

4. Alonso PL, Brown G, Arevalo-Herrera M, Binka F, Chitnis C, Collins F, et al. A research agenda to underpin malaria eradication. PLoS Med. 20118:e1000406.

5. Malcolm CA, El Sayed B, Babiker A, Girod R, Fontenille D, Knols BGJ, et al. Field site selection: getting it right first time around. Malar J. 2009;8(Suppl 2):S9.

6. Alphey N, Coleman PG, Donnelly CA, Alphey L. Managing insecticide resistance by mass release of engineered insects. J Econ Entomol. 2007;100:1642-9.

7. Alphey L, Benedict M, Bellini R, Clark GG, Dame DA, Service MW, et al. Sterile-insect methods for control of mosquito-borne diseases: an analysis. Vector Borne Zoonotic Dis. 2010;10:295-311.

8. Knipling EF. Possibilities of insect control or eradication through the use of sexually sterile males. J Econ Entomol. 1955;48:459-62.

9. Tripet F, Touré YT, Dolo G, Lanzaro GC. Frequency of multiple inseminations in field-collected Anopheles gambiae females revealed by DNA analysis of transferred sperm. Am J Trop Med Hyg. 2003;68:1-5.

10. Charlwood JD, Jones MDR. Mating behaviour in the mosquito, Anopheles gambiae s.l. I. close range and contact behavior. Physiol Entomol. 1979;2: $111-20$.

11. Vreysen MJ, Saleh KM, Ali MY, Abdulla AM, Zhu ZR, Juma KG, et al. Glossina austeni, (Diptera : Glossinidae) eradicated on the Island of Unguja, Zanzibar, using the sterile insect technique. J Econ Entomol. 2000;93:123-135.

12. Dantas L, Pereira R, Silva N, Rodrigues A, Costa R. The SIT control programme against Medfly on Madeira Island. In: Proceedings of the 6th International Symposium on fruit flies of economic importance. South Africa: Stellenbosch; 2002.

13. Koyama J, Kakinohana H, Miyatake T. Eradication of the melon fly, Bactrocera cucurbitae, in Japan: Importance of behavior, ecology, genetics, and evolution. Annu Rev Entomol. 2004;49:331-49.

14. Krafsur ES, Whitten CJ, Novy JE. Screwworm eradication in North and Central America. Parasitol Today. 1987;3:131-7.

15. Benedict $M Q$, Robinson AS. The first releases of transgenic mosquitoes: an argument for the sterile insect technique. Trends Parasitol. 2003;19: 349-55.

16. Franz G, Robinson AS. Molecular technologies to improve the effectiveness of the sterile insect technique. Genetica. 2011;139:1-5.

17. Nolan T, Papathanos P, Windbichler N, Magnusson K, Benton J, Catteruccia F, et al. Developing transgenic Anopheles mosquitoes for the sterile insect technique. Genetica. 2011;139:33-9.

18. Alphey L, Beard CB, Billingsley P, Coetzee M, Crisanti A, Curtis C, et al. Malaria control with genetically manipulated insect vectors. Science. 2002;298:119-21.

19. Alphey L, Nimmo D, O'Connell S, Alphey N. Insect population suppression using engineered insects. Adv Exp Med Biol. 2008;627: 93-103.

20. Thomas D, Donnelly C, Wood R, Alphey L. Insect population control using a dominant, repressible, lethal genetic system. Science. 2000;287:2474-6.

21. Heinrich JC, Scott MJ. A repressible female-specific lethal genetic system for making transgenic insect strains suitable for a sterile-release program. Proc Natl Acad Sci USA. 2000;97:8229-32. 
22. Phuc HK, Andreasen MH, Burton RS, Vass C, Epton MJ, Pape G, et al. Late-acting dominant lethal genetic systems and mosquito control. BMC Biol. 2007:5:11.

23. Bargielowski I, Nimmo D, Alphey L, Koella JC. Comparison of life history characteristics of the genetically modified OX513A line and a wild type strain of Aedes aegypti. PLoS One. 2011;e20699:6.

24. Fu G, Lees RS, Nimmo D, Aw D, Jin L, Gray P, et al. Female-specific flightless phenotype for mosquito control. Proc Natl Acad Sci U S A. 2010;107:4550-4.

25. Wise de Valdez MR, Nimmo D, Betz J, Gong HF, James AA, Alphey, L, et al. Genetic elimination of dengue vector mosquitoes. Proc Natl Acad Sci U S A. 2011;108:4772-5.

26. Harris AF, Nimmo D, McKemey AR, Kelly N, Scaife S, Donnelly CA, et al. Field performance of engineered male mosquitoes. Nat Biotechnol. 2011:29:1034-7.

27. Harris A, McKemey A, Nimmo D, Curtis Z, Black I, Morgan S, et al. Successful suppression of a field mosquito population by sustained release of engineered male mosquitoes. Nat Biotechnol. 2012;30:828-30.

28. Lacroix R, McKemey AR, Raduan N, Kwee Wee L, Hong Ming W, Guat Ney $T$, et al. Open field release of genetically engineered sterile male Aedes aegypti in Malaysia. PLoS One. 2012;e42771:7.

29. Howell PI, Knols BG. Male mating biology. Malar J. 2009;8(Suppl 2):1-10.

30. Mayer DG, Atzeni MG, Stuart MA, Anaman KA, Butler DG. Mating competitiveness of irradiated flies for screwworm fly eradication campaigns. Prev Vet Med. 1998;36:1-9.

31. Andreasen $\mathrm{MH}$, Curtis CF. Optimal life stage for radiation sterilization of Anopheles males and their fitness for release. Med Vet Entomol. 2005;19: 238-44.

32. Catteruccia F, Benton JP, Crisanti A. An Anopheles transgenic sexing strain for vector control. Nat Biotechnol. 2005;23:1414-7.

33. Howell P, Benedict MQ. Mating competitiveness of Anopheles arabiensis males as a function of transgenic state and genetic similarity to females. J Insect Behav. 2009;22:477-91.

34. Lee HL, Vasan S, Ahmad NW, Idris I, Hanum N, Selvi S, et al. Mating compatibility and competitiveness of transgenic and wild type Aedes aegypti; (L.) under contained semi-field conditions. Transgenic Res. 2013;22:47-57.

35. Klein TA, Windbichler N, Deredec A, Burt A. Benedict MQ. Infertility resulting from transgenic I-Ppol male Anopheles gambiae, in large cage trials. Pathog Glob Health. 2012;106:20-31.

36. Windbichler N, Papathanos PA, Crisanti A. Targeting the X chromosome during spermatogenesis induces $Y$ chromosome transmission ratio distortion and early dominant embryo lethality in Anopheles gambiae. PLoS Genet. 2008;4:e1000291.

37. Thailayil J, Magnusson K, Godfray CJH, Crisanti A, Catteruccia F. Spermless males elicit large-scale female responses to mating in the malaria mosquito Anopheles gambiae. Proc Natl Acad Sci U S A. 2011;108: 13677-81.

38. Foster GG, Vogt WG, Woodburn TL, Smith PH. Computer simulation of genetic control. Comparison of sterile males and field-killing female solutions. Theor Appl Genet. 1988;76:870-9.

39. Schliekelman P, Gould F. Pest control by the introduction of a conditional lethal trait on multiple loci: Potential, limitations, and optimal strategies. J Econ Entomol. 2000;93:1543-65.

40. Schliekelman P, Gould F. Pest control by the release of insects carrying a female-killing allele on multiple loci. J Econ Entomol. 2000;93:1566-79.

41. Barclay HJ. Modeling incomplete sterility in a sterile release program: interactions with other factors. Popul Ecol. 2001;43:197-206.

42. Esteva L, Yang HM. Mathematical model to assess the control of Aedes aegypti mosquitoes by the sterile insect technique. Math Biosci. 2005;198: $132-47$.

43. Dye C. Models for the population-dynamics of the yellow-fever mosquito, Aedes aegypti. J Anim Ecol. 1984;53:247-68.

44. Kean JM, Wee SL, Stephens AEA, Suckling DM. Modelling the effects of inherited sterility for the application of the sterile insect technique. Agric Forest Entomol. 2008:10:101-10.

45. Yakob L, Bonsall MB. Importance of Space and Competition in Optimizing Genetic Control Strategies. Biol Microb Control. 2009;102:50-7.

46. White SM, Rohani P, Sait SM. Modelling pulsed releases for sterile insect techniques: fitness costs of sterile and transgenic males and the effects on mosquito dynamics. J Appl Ecol. 2010;47:1329-39.
47. Deredec A, Godfray CJH, Burt A. Requirements for effective malaria control with homing endonuclease genes. Proc Natl Acad Sci USA. 2011;108:E874-80.

48. Dumont $Y$, Tchuenche JM. Mathematical studies on the sterile insect technique for the Chikungunya disease and Aedes albopictus. J Math Biol. 2011;65:1-46.

49. Lee SS, Baker RE, Gaffney EA, White SM. Modelling Aedes aegypti mosquito control via transgenic and sterile insect techniques: Endemics and emerging outbreaks. J Theor Biol. 2013;331:78-90.

50. Arifin SM, Zhou Y, Davis G, Gentile JE, Madey GR, Collins FH. An agent-based model of the population dynamics of Anopheles gambiae. Malar J. 2014;13:424

51. Yaro AS, Dao A, Adamou A, Crawford JE, Ribeiro JMC, Gwadz R, et al. The distribution of hatching time in Anopheles gambiae. Malar J. 2006;5:19.

52. Styer LM, Carey JR, Wang JL, Scott TW. Mosquitoes do senesce: departure from the paradigm of constant mortality. Am J Trop Med Hyg. 2007;76:111

53. Yakob L, Alphey L, Bonsall MB. Aedes aegypti control: the concomitant role of competition, space and transgenic technologies. J Appl Ecol. 2008;45:1258-1265.

\section{Submit your next manuscript to BioMed Central and take full advantage of:}

- Convenient online submission

- Thorough peer review

- No space constraints or color figure charges

- Immediate publication on acceptance

- Inclusion in PubMed, CAS, Scopus and Google Scholar

- Research which is freely available for redistribution

Submit your manuscript at www.biomedcentral.com/submit
C BioMed Centra 